\title{
Percepción frente a la obesidad en padres y adolescentes pertenecientes a la ciudad de Armenia, Quindío
}

Perception of obesity among parents and adolescents from the city of Armenia, Quindío

Percepção da obesidade em pais e adolescentes da cidade de Armênia, Quindío

Vivian Estefhany Acevedo, Enf..$^{1} \mathbb{D}$, Carolina Palacios-Recalde, Enf. ${ }^{1}(\mathbb{D}$, María Fernanda Villamil-Pineda, Enf. ${ }^{1}$ (D)

1. Enfermera, Corporación Universitaria Empresarial Alexander Von Humboldt. Armenia, Quindío, Colombia.

Correspondencia. María Fernanda Villamil Pineda. Corporación Universitaria Empresarial Alexander Von Humboldt. Barrio nuevo Armenia Manzana D casa 1 etapa 1. Email: nanda0196@gmail.com

\section{INFORMACIÓN DEL ARTÍCULO:}

Artículo recibido: 18 de febrero de 2019

Artículo aceptado: 21 de enero de 2020

DOI: https://doi.org/10.29375/01237047.3514

Cómo citar: Acevedo VE, Palacios-Recalde C, Villamil-Pineda MF. Percepción frente a la obesidad en padres y adolescentes pertenecientes a la ciudad de Armenia, Quindío. MedUNAB. 2020;23(2): 270-280. doi: $10.29375 / 01237047.3514$

\section{RESUMEN}

Introducción. La obesidad es una enfermedad de interés para la salud pública, con alto índice de prevalencia en el mundo, la cual trae consigo problemas de salud que ponen en riesgo la vida de las personas que la padecen. El estado nutricional de un menor está determinado por características sociales, culturales y económicas que se pueden ver influenciadas por las costumbres arraigadas durante la niñez. De acuerdo con lo anterior, la presente investigación tiene como objetivo conocer las creencias y hábitos que tienen los adolescentes y sus padres frente a la obesidad en la ciudad de Armenia, Quindío. Metodología. Investigación cualitativa de enfoque 
fenomenológico, realizada por medio de entrevistas semiestructuradas en la que participaron 7 padres y 7 adolescentes. Resultados. Según los padres, la mayoría de adolescentes invierten su tiempo en aparatos electrónicos, dejando a un lado la realización de ejercicio y los estilos de vida saludable. Un participante consideró a los padres o tutores encargados del cuidado de los menores como responsables de la alimentación. Discusión. En las percepciones de los padres y los adolescentes frente al tema de la obesidad se encontraron coincidencias en las diferentes respuestas de ambos grupos. Conclusión. Los adolescentes se encuentran con libertad para acceder a tecnologías virtuales, tanto en sus colegios como en sus hogares; sin embargo, según la literatura, el juego físico posee un papel fundamental en el desarrollo del niño, así como el papel de los padres dentro del desarrollo nutricional de los menores, el cual es factor determinante, puesto que el comportamiento de los niños suele modelarse de la observación y la adaptación.

Palabras claves:

Adolescente; Padres; Conducta Alimentaria; Obesidad; Estilo de vida; Investigación Cualitativa.

\section{ABSTRACT}

Introduction. Obesity is a disease of interest for public health with a high rate of prevalence in the world, which brings health problems that put at risk the lives of those who suffer from the disease. The nutritional status of children is determined by social, cultural and economic characteristics that may be influenced by habits acquired during childhood. According to the above, the present research aims to find out the beliefs and habits of adolescents and parents regarding obesity in the city of Armenia, Quindío. Methodology. Qualitative research with a phenomenological approach, conducted through semi-structured interviews with seven parents and seven adolescents. Results. According to the parents, most adolescents spend their time on electronic devices, forgetting about exercising and healthy lifestyles. One participant thought that children's parents or guardians are responsible for their diet. Discussion. Similarities in perceptions of obesity were found between the parents and adolescents in the answers of both groups. Conclusion. Adolescents are free to access digital technology in both their schools and homes. However, according to the literature, physical play has an essential role in child development, as well as the role of parents in children's nutritional development. This is a determining factor as children's behavior is usually modeled through observation and adaptation.

Keywords:

Adolescent; Parents; Feeding Behavior; Obesity; Life Style; Qualitative Research.

\section{RESUMO}

Introdução. A obesidade é uma doença de interesse para a saúde pública, com alta taxa de prevalência no mundo, o que traz consigo problemas de saúde que coloca em risco a vida de quem a sofre. O estado nutricional de uma criança é determinado por características sociais, culturais e econômicas que podem ser influenciadas por costumes arraigados durante a infância. De acordo com o exposto, a presente pesquisa tem como objetivo conhecer as crenças e hábitos que os adolescentes e seus pais têm em relação à obesidade na cidade de Armênia, Quindío. Metodologia. Pesquisa qualitativa, com abordagem fenomenológica, realizada por meio de entrevistas semiestruturadas, na que participaram sete pais e sete adolescentes. Resultados. Segundo os pais, a maioria dos adolescentes gasta seu tempo com dispositivos eletrônicos, deixando de lado a realização de atividade física e estilos de vida saudáveis. Um participante considerou os pais ou tutores de menores como responsáveis pela alimentação. Discussão. Nas percepções de pais e adolescentes sobre a questão da obesidade, foram encontradas coincidências nas diferentes respostas dos dois grupos. Conclusão. Os adolescentes têm a liberdade para acessar tecnologias virtuais, tanto em suas escolas quanto em suas casas. No entanto, de acordo com a literatura, os jogos físicos têm papel fundamental no desenvolvimento da criança, assim como o papel dos pais no desenvolvimento nutricional de menores, o que é um fator determinante, uma vez que o comportamento da criança costuma ser modelado a partir da observação e adaptação.

Palavras-chave:

Adolescente; Pais; Comportamento alimentar; Obesidade; Estilo de vida; Pesquisa qualitativa. 


\section{Introducción}

Según la Organización Mundial de la Salud (OMS), la obesidad es una enfermedad crónica de interés en salud pública, constituye un problema grave, caracterizado por acumulación excesiva de grasa que afecta negativamente la vida de la persona que la padece. Esta patología puede ser de origen multifactorial; sin embargo, la mayoría de personas obesas atribuyen su condición a la falta de actividad física y al sedentarismo prolongado. Con base en la Encuesta Nacional de Situación Nutricional "uno de cada 6 niños y adolescentes presenta sobrepeso $u$ obesidad en Colombia; esta relación aumenta a medida que se incrementa el nivel del SISBEN y el nivel educativo de la madre". Además, el exceso de peso en individuos es mayor en el área urbana que en la rural, siendo de $19.2 \%$ y $13.4 \%$, respectivamente $(1,2)$.

Estas cifras toman gran relevancia por investigaciones como la de Escudero, Morales, et al., quienes exponen que la obesidad general y la adiposidad visceral se asocian con un mayor riesgo cardiovascular y metabólico; específicamente, la principal consecuencia de la obesidad infantil es la permanencia en las siguientes esferas del ser humano, y el desencadenamiento de patologías crónicas como hipertensión, diabetes mellitus e hiperlipoproteinemia (3).

En el marco del desarrollo humano, la infancia y la adolescencia son etapas fundamentales para la formación y crecimiento del individuo, por lo cual, los niños deben recibir una alimentación correcta en cantidad y calidad (4). La alimentación, como factor estructural, ha presentado en las últimas décadas cambios en sus patrones, asociados a las transformaciones sociales y demográficas que han sustituido la alimentación tradicional; principalmente se han cambiado alimentos de origen vegetal (cereales integrales, frutas, verduras de hoja, legumbres, frutos oleaginosos, raíces y tubérculos) por alimentos de alta densidad energética, los cuales incluyen alimentos de elevado contenido en grasa total y grasas saturadas, azúcares refinados, refrescos y cereales. Asimismo, se conoce que la población consume niveles de sal mucho más elevados que los recomendados para la prevención de enfermedades y que a nivel mundial, los más afectados por este fenómeno son las poblaciones menos favorecidas que optan por opciones de alimentación más 'baratas' y, en general, son las menos saludables (5).

La dieta es un factor clave para la prevención de las Enfermedades No Transmisibles (ENT), por lo que mantener una mala alimentación aumenta la probabilidad de perpetuar las ENT en las poblaciones, generando riesgo de aumentar la presión arterial, la glucosa, los lípidos en la sangre y la obesidad. Este conjunto se conoce como factores de riesgo metabólicos que afectan a todos los grupos de edad y se suelen asociar a los grupos de edad más avanzada; aunque los riesgos asociados a las dietas malsanas comienzan en la niñez y se acumulan a lo largo de la vida (6).

Son numerosas las consecuencias negativas para la salud que la obesidad produce en niños y adolescentes; se puede destacar que la obesidad trae consigo problemas de tipo psicológico, sociales, cardio respiratorios, ortopédicos, endocrinos y dermatológicos (7).

Sumado a lo anterior, el $60 \%$ de los niños que están con sobrepeso en el período prepuberal y $80 \%$ de los que lo están en la adolescencia, estarán con sobrepeso en la adultez temprana $(8,9)$. Sin embargo, esta enfermedad en infantes está aumentando de forma exponencial, tanto en países en desarrollo como en países desarrollados (10).

Teniendo en cuenta lo anterior, el sobrepeso y la obesidad tiene un origen multifactorial en el que se involucran la susceptibilidad genética, los estilos de vida y las características del entorno, con influencia de diversos determinantes subyacentes, como la globalización, la cultura, la condición económica, la educación, la urbanización y el entorno político y social que generan cambios en los estilos de vida saludables de los grupos (11).

Paralelo a la regulación alimenticia, resulta imprescindible la práctica de actividad física adecuada, en la que el individuo se sienta a gusto y pueda realizarla de forma sistemática, al menos tres veces por semana y durante 30 minutos como mínimo. A su vez, es importante incentivar la práctica de actividad física no programada en cualquier grupo de edad, algunas opciones son subir escaleras en lugar de utilizar el ascensor o elementos mecánicos; ir a pie para desplazarse de un lugar a otro; montar en bicicleta, jugar al fútbol, jugar con cuerdas, etc. A pesar de que el gasto energético en estas actividades pueda resultar pequeño en un primer momento, su continuidad a lo largo de meses y años puede significar la diferencia entre tener o no una condición de obeso. También se debe considerar que las personas que ya padecen de obesidad, especialmente en la niñez y adolescencia, pueden presentar perjuicios en el desarrollo psicosocial y bienestar general, debido a ataques de otros o de sí mismo, por su figura y comportamientos asociados a ella, como la pereza o falta de preocupación personal (12). 
Una investigación relacionada con este tema es la expuesta por Núñez Rivas, en la cual se indica que: El niño y la niña elaboran distintas representaciones sociales sobre la obesidad, de acuerdo con la influencia (formas de pensar, creencias construidas, etc.) de sus pares, familiares y otras fuentes con las que están en contacto cotidianamente tanto de manera directa como indirecta. (13)

De forma análoga, otros investigadores han afirmado: El niño y la niña nacen en el seno de un grupo familiar, dentro de un país específico y en un momento socio histórico determinado, por lo tanto, están socialmente predispuestos a adoptar cierto tipo de representaciones sociales y creencias prefijadas (14).

Debido a las pocas investigaciones que se han realizado sobre la percepción de los padres frente a la obesidad, surge la necesidad de conocer las creencias y hábitos que hacen parte de cada persona durante su infancia, ya que los padres son el principal enfoque de representación de ejemplo para los niños.

La OMS ha creado el Plan de acción mundial para la prevención y el control de las enfermedades no transmisibles 2013 - 2020, que tiene por objeto cumplir los compromisos de la Declaración Política de las Naciones Unidas sobre las Enfermedades No Transmisibles. (15); este plan se basa en la existencia de reportes en donde se muestra que la mayoría de la población mundial vive en países donde el sobrepeso y la obesidad cobran más vidas de personas que la insuficiencia ponderal (1).

Por otro lado, se debe considerar que las cifras nombradas son producto de tradiciones arraigadas en torno a la ingesta de alimentos, cuya base es susceptible de ser cuestionada a través de procesos de socio educación, creencias y hábitos, adicionando, como ya se estableció, la indiscutible relación con cuestiones familiares y psicológicas, sin olvidar hacer conciencia de los problemas que acarrea la obesidad en todos los sentidos (16).

Según lo indica la OMS, desde 1980 "la obesidad se ha más que doblado en todo el mundo, en el año 2014, más de 1,900 millones de adultos de 18 o más años tenían sobrepeso, de los cuales, más de 600 millones eran obesos. Así mismo en este año, el 39\% de las personas adultas de 18 o más años tenían sobrepeso, y el 13\% eran obesas". Todo lo anterior, demuestra que la obesidad infantil es uno de los problemas de salud pública graves del siglo XXI. La prevalencia ha aumentado a un ritmo alarmante. Se calcula que en el 2016, más de 41 millones de niños menores de cinco años en todo el mundo tenían sobrepeso o eran obesos (1)

Durante la niñez y la adolescencia, las costumbres y creencias son quienes definen los hábitos del adulto; por tal razón, se realizó esta investigación con el objetivo de conocer la percepción frente a la obesidad en los adolescentes y sus padres de cara a la obesidad en la ciudad de Armenia, Quindío.

\section{Metodología}

Se realizó un estudio cualitativo con diseño fenomenológico, el cual se enfocó en el comportamiento según las experiencias y percepciones individuales, y aspectos subjetivos de los participantes, se buscaron conceptos, creencias y sentimientos que tenían los adolescentes y sus padres acerca de la obesidad. La información se recolectó a través de una entrevista semiestructurada, realizada por medio de muestra llamada "casos - tipo", la cual se constituye por casos que reúnen características típicas como la edad, grado de escolarización, sexo, estado civil, ocupación, sector de residencia y personas con las que comparten vivienda.

El análisis se realizó en tres categorías: conocimientos, creencias y sentimientos, determinadas durante la inclusión de la literatura de Neisser sobre la percepción, la entrevista inició con la prueba piloto a un padre y un adolescente, inicialmente con un número de 15 preguntas, esto con el fin de analizar las falencias y modificar las preguntas para los siguientes participantes; además de analizar la efectividad de las categorías propuestas basadas en la psicología clásica de Neisser sobre la percepción, se aplicó la entrevista hasta saturar información por medio de validación y repetición de resultados de cada uno de los participantes, con un estudio de riesgo bajo.

Finalmente, la entrevista se basó en 8 preguntas, las cuales estaban distribuidas según la categoría, donde la categoría conocimiento contaba con 4 preguntas, emociones con 2 preguntas y sentimientos con 2 preguntas, permitiendo cada una dar cumplimiento al objetivo de esta investigación. El entrevistador tenía la libertad de indagar más acerca de sus respuestas para obtener mayor información. La población de estudio se tomó por muestreo intencional con fácil accesibilidad; para el tipo de estudio se tuvo en cuenta un grupo de enfoque de siete casos por grupo (siete adolescentes y siete padres), la selección de los participantes se realizó de forma aleatoria simple, y los criterios de inclusión fueron adolescente de sexo masculino y/o femenino que comprendiera la etapa de adolescencia tardía 
según la OMS, entre 14 y 19 años, hijo biológico del padre a entrevistar, sin ningún otro tipo de inclusión o exclusión en sus determinantes sociales; madre o padre legal y biológico del adolescente a entrevistar. Se aplicó consentimiento informado previamente aprobado por el Comité de Ética de Investigación de la Facultad de Ciencias de la Salud de la Corporación Universitaria Empresarial Alexander Von Humboldt, Armenia, Quindío.

Se informó a los participantes de la propuesta de investigación, estableciendo aspectos éticos de participación voluntaria y de confidencialidad, aclarados anteriormente en el consentimiento informado, previo a la entrevista. Se inició el acercamiento realizando visitas domiciliarias a cada uno de los participantes donde dos de las tres investigadoras tomaron 4 participantes cada una, la tercera tomó 6 participantes por facilidad de acercamiento al grupo focal, cada una de las investigadoras contaba con un diario de campo; la información fue recolectada a través de notas de grabación del dispositivo móvil de cada investigador, se transcribió a un formato de análisis el cual permitió la distribución de las categorías donde se agruparon las respuestas similares y se identificaron las diferentes respuestas para comparar con estudios semejantes a la investigación.

Para los criterios de validez se tuvo en cuenta la confiabilidad de las respuestas contada por los participantes en cada una de las preguntas de las diferentes categorías frente a la percepción que cada uno tenía acerca de la obesidad, las preguntas de cada categoría: conocimientos, emociones y sentimientos permitieron alcanzar la mayor cantidad de información hasta la saturación de datos. La entrevista se realizó por notas de grabación, transcritas a un formato de análisis para lograr así una confirmabilidad.

\section{Resultados}

Adolescentes: en general, los adolescentes definen la obesidad como el aumento de peso por fuera de los rangos normales, el cual afecta la imagen corporal. En esta investigación las causas identificadas que llevaron al sobrepeso y obesidad fueron los hábitos de origen físicos o alimentarios, en donde se considera que la razón de este estado se debe al aumento de la ingesta de comida chatarra, y a la cantidad de porciones de más que una persona come. Por ejemplo, A2 respondió: "Porque comen mucho, comen 10 porciones, no sé, o más. Porque comen comida chatarra como hamburguesas, cosas que tienen grasas".
Además, los participantes relacionaron este aumento de raciones con la ansiedad o el sentimiento de tristeza que la persona puede experimentar. Así lo mencionó A1: "Porque de pronto pueden tener ansiedad o pueden estar tristes y su solución es comer".

Sin embargo, es importante considerar que otra parte de los adolescentes atribuyen el sobrepeso y obesidad al sedentarismo relacionado con la falta de actividad física. En este sentido, A6 expresó: "Por la mala alimentación, por no hacer ejercicio, por dormir tanto"; y A5 dijo: "Porque no se cuidan y porque no hacen ejercicio y tienden a comer más comida chatarra, que la de sal".

Los entrevistados argumentan que el aumento de peso es generado por el estilo de vida, las personas con sobrepeso y obesidad generan unas condiciones donde resulta comprometido su bienestar emocional y físico, presentando síntomas como dolores articulares y problemas cardíacos. De manera textual, A5 expuso: "Las complicaciones que puede generar la obesidad son dolor de espalda o de rodillas porque al cargar más peso que los demás que de lo que puede cargar su espalda se complican". Asimismo, A4 menciona: "infarto, ser rechazado por las personas, no sé, tal vez, sufrir problemas de la presión".

En cuanto a la socialización de las personas obesas, para los adolescentes esta se ve afectada por varios componentes, los cuales pueden finalizar en prácticas como la discriminación, rechazo, bullying y depresión. Por ejemplo, A4 dijo: "creo que es muy difícil para ellos porque las personas las discriminan y los ven objetos de discriminación por ser diferentes a ellos".

Es de resaltar que, para los adolescentes el sentimiento que estas personas les generan es nobleza y humildad, de lo cual se podría deducir que esta forma de pensamiento hace que las personas obesas tengan mejor aceptación en la sociedad.

Padres: ellos definen la obesidad como alteraciones a nivel metabólico, catalogándola como un desorden hormonal que conlleva a una cantidad de factores que deterioran el organismo. En este contexto, P1 opinó: "Bueno, yo pienso que en Colombia la obesidad ya es una enfermedad pública, porque no solamente se está dando en personas adultas o mayores, sino que se está dando en la población infantil, la obesidad es un desorden hormonal que conlleva a una cantidad de factores que deterioran el organismo del ser humano conllevándolo al sobrepeso". 
Se destaca el concepto de obesidad de un padre, ya que para él está asociado con desorganización en cuanto a sus hábitos alimenticios y físicos. Así, P4 definió: "La obesidad para mi es una persona desorganizada, mantiene comiendo mucho y no hace nada, desorganización porque uno tiene que ser organizado para todo hasta para el estado de uno corporalmente".

Para los padres, la mayoría de adolescentes invierten su tiempo en aparatos electrónicos dejando de lado la realización de ejercicio. La mayoría de los padres atribuyen que el sobrepeso y la obesidad son generados por la falta de actividad física y los malos hábitos alimenticios; sin embargo, ninguno de ellos se refirió a qué son los hábitos saludables. Así, P1 piensa: "Bueno, algunos se vuelven porque pues no cuentan con buenos hábitos alimenticios, algunos son hereditarios y hay un flagelo que está afectando no solamente a los jóvenes sino también a la juventud en general que son los electrónicos todo lo que son computadores, celulares, tabletas hacen que los niños hoy en día o las personas se vuelvan muy sedentarios".

La mayoría de los padres coincidieron que la obesidad trae consigo problemas a nivel sistémico, el cual relacionan con el cansancio y fatiga. Por ejemplo, P2 comentó: "la fatiga, van a tener problemas de cansancio, de demora en hacer alguna actividad. No se va a desenvolver muy bien en algún ámbito físico".
A nivel económico y emocional, los padres consideran que una persona de talla grande sufre discriminaciones, rechazo y, en caso de ser niños, pueden sufrir bullying por parte de sus compañeros de colegio. Así como lo expresó P1: "Bueno, hay varios, hay socioeconómicos, emocionales y se pueden incluir también el aspecto personal porque una persona de talla grande llamémoslo así, la sociedad muchas veces lo margina, es rechazado, se puede ver en el colegio con los niños que se hacen demasiado bullying".

Acerca de lo que piensan de una persona con sobrepeso u obesidad, según P7, es falta de confianza, explica que no le genera una buena percepción debido a que su apariencia física no se encuentra cuidada, por esto, él considera que no es una persona apta para generar un buen impacto en la sociedad.

Solo un participante consideró como responsables de la alimentación a los padres o tutores encargados del cuidado de los menores. Esto ocurrió cuando P1 opinó: "Poco acompañamiento o interés por los padres o tutores responsables del menor, de pronto por las actividades laborales o porque viven en ciudades donde son muy congestionadas y el desplazarse de un lugar a otro acarrea demasiado tiempo".

Tabla 1. Paralelo de padres y adolescentes de cada categoría

\section{Conocimiento}

\section{Padres:}

Los padres definieron la obesidad como problemas, o alteraciones, tanto a nivel metabólico como desórdenes alimenticios y hábitos, en cuanto a alteraciones metabólicas: P1'La obesidad es un desorden hormonal que conlleva una cantidad de factores que deteriora el organismo del ser humano conllevándolo al sobrepeso".

Solo uno de ellos encaminó el concepto hacia hábitos: P4”La obesidad para mi es una persona desorganizada, mantiene comiendo mucho y no hace nada, desorganización porque uno tiene que ser ordenado para todo hasta para el estado de uno corporalmente".

En lo que se refiere a las causas, los padres consideraron principalmente como causas de la obesidad los hábitos de conducta física, reiterando el sedentarismo, así lo refirieron los participantes: P1"Bueno, algunos se vuelven porque pues no cuentan con buenos hábitos alimenticios, algunos son hereditarios y hay un flagelo que está afectando no solamente a los adultos sino también a la juventud en general, que son los electrónicos, todo lo que son computadores, celulares, tabletas, hacen que los niños hoy en día o las personas se vuelvan muy sedentarios.

\section{Adolescentes:}

Según los adolescentes, la obesidad la definen como un aumento de peso considerable en una persona que lo refleja físicamente. A2 "Es cuando la persona está muy gorda y no puede caminar, gorda es una persona que no cabe por un espacio. A4 "Es una persona que está pasada de peso"

Para los adolescentes, las principales causas de la obesidad son hábitos comportamentales alimentarios o físicos, lo cual genera consecuencias a nivel físico y psicológico. A1: "De pronto por el peso que se acumula en la barriga, puede dañar la espalda, como encorvarla, afecta las rodillas por el peso que no pueden soportar las rodillas y el corazón".

Y de esta forma la mayoría de los participantes coincidieron en que las principales formas de prevenir la obesidad son los estilos de vida saludable, como la práctica de actividad física y una alimentación balanceada: A1: "Haciendo ejercicio, dejando de comer grasas saturadas". 


\section{Creencias}

\section{Padres:}

Con respecto al pensamiento de los padres hacia una persona obesa, todos respondieron de manera diferente, teniendo en cuenta que algunos de los mismos refirieron tener pensamientos negativos hacia estas personas. P2 "No, que de pronto están mal orientados o el problema también es que la gente piensa generalmente que el que come harto es saludable y resulta que no es así, comer harto no quiere decir que uno coma bien, comer bien es comer lo adecuado, solo lo esencial".

$\mathrm{Y}$ dos de ellos consideraron que las personas obesas no son culpables de su condición ya que esto se les presentó desde su nacimiento, o en el desarrollo de alguna enfermedad. P6 "Pues, la verdad en sí uno a veces no puede criticar, porque como te digo, que si de pronto tiene la enfermedad es porque nació con ella". En cuanto a cómo creían los padres que era la socialización de las personas obesas con los demás, la mayoría de los participantes refirieron creer que la socialización de las personas se veía afectada negativamente, debido a su condición. Así lo indican: P1 "Bueno, la socialización es un poco difícil porque de pronto socializar con el grupo familiar, pero ya al exterior son estigmatizados, mucha gente los ve y se burlan, los critican y eso directa o indirectamente afecta en el subconsciente del ser humano".

Sin embargo, solo un participante expresó tener una opinión positiva en cuanto a la socialización de las personas obesas, refiriendo que son personas que aparentan ser felices: p3 "Pues, por lo que he visto, creo que es normal, incluso de pronto aquí las personas que son obsesas pues, no sé si sea así, pero uno los ve felices normalmente"

Solo un participante consideró como responsables de la inadecuada alimentación a los padres o tutores encargados del cuidado de los menores, razón por la cual se presenta la obesidad en los mismos y de esta manera justificó su respuesta:

P1 "Poco acompañamiento o interés por los padres o tutores responsables del menor, de pronto por las actividades laborales o porque viven en ciudades donde son muy congestionadas y el desplazarse de un lugar a otro acarrea demasiado tiempo.

\section{Adolescentes:}

En cuanto a qué pensaban los adolescentes sobre una persona obesa, se pudieron evidenciar diferentes respuestas por parte de los mismos, teniendo en cuenta que algunos lo ven como problemas, alimentación inadecuada, falta de cuidado; al contrario de dos participantes quienes consideraron que las personas obesas totalmente normales. A1 "Es una persona que tiene problemas, que lo solucionan comiendo, puede ser como que no es capaz de autorregularse".

Con respecto a la socialización de las personas obesas con los demás, la mayoría de los adolescentes entrevistados consideraron que la socialización era difícil por varios factores, dentro de ellos el rechazo por parte de la sociedad, la discriminación y el bullying que se presenta cada vez más, así lo refirieron: A1 "No es normal, creo que es complicado ya que ellos buscan aceptación entonces es como difícil hablar con los familiares, ya que algunos no entienden su problema y los comienzan a criticar".

Sin embargo, solo un participante consideró la socialización de las personas obesas con los demás como una relación normal: A3 "Pues yo creo que, de forma normal, yo tengo varios amigos obesos y se relacionan normal, como si nada" 


\section{Sentimientos}

\section{Padres:}

Para los padres, ver a una persona obesa genera diferentes sentimientos; algunos positivos y otros negativos. P1 "Bueno, es que todo gordito como se dice de corazón, genera como nobleza, ternura". P7 "Depresión, de que son incapaces por ejemplo hasta de caminar. A mí me da sentimiento ver una persona obesa porque igual ya las cosas no le quedan, o sea, por ejemplo, en el sentido de la ropa ya no le queda igual como antes, ya uno no se ve de una forma agradable, ya uno no agrada por el motivo de esta enfermedad".

Aunque para otros padres, una persona obesa no les generaba ningún sentimiento, ya que para ellos son personas normales y no los ven como menos o más que los demás. P2 "La verdad, ninguno".

Fuente: Elaborada por los autores

\section{Discusión}

Los relatos de los participantes permitieron contrastar la percepción que tenían frente a la obesidad cotejada con algunos estudios como se muestra a continuación.

\section{Conocimiento}

Para los padres, la obesidad es consecuencia de una inadecuada alimentación y, a pesar de ser los cuidadores, no se consideraron como responsables de esta. Apoyando esta afirmación, Cabello Garza y de Jesús, explican que "las madres no solo perciben de manera distorsionada la imagen corporal de su hijo, sino que lo justifican y no asumen la enfermedad por lo que se ubican ajenas a que su hijo padezca un problema de salud". Además, Olivares, Bustos, et al., afirman que las madres no mostraron una actitud de apoyo para intentar controlar o revertir la situación de obesidad en su hijo/a obeso/a en un $98.8 \%$ de los casos que estudiaron $(17,18)$.

No obstante, Rodríguez, Ruíz et al., encontraron en su estudio que los progenitores percibieron el problema del sobrepeso y la obesidad de sus hijos en más de la tercera parte de los casos, en especial en las niñas. Del mismo modo que su responsabilidad en la alimentación. El manejo e identificación de estas afecciones son importantes, ya que algunos factores implicados en la obesidad infantil son la percepción parental inadecuada del estado nutricional de los hijos; situación que, según

\section{Adolescentes:}

Para los adolescentes entrevistados, ver a una persona obesa les genera diferentes conmociones, en este caso, la mayoría de los expresaron sentimientos negativos, como tristeza, pesar y depresión. Así lo indicaron: A2 "tristeza". A4 "Mmmm, me genera pesar".

Sin embargo, dos de ellos se expresaron de manera diferente, para uno de ellos, ver a una persona obesa le generaba sentimiento positivo. A1: "Nobleza". Y, al contrario, el otro participante conserva una despreocupación frente a la persona obesa, ya que no se trata de él mismo. A6 "Normal, pues sí porque como no es uno, es la otra persona, entonces..." los padres entrevistados en la presente investigación, podría deberse a las actividades laborales o congestión de las ciudades donde habitan, produciendo el poco acompañamiento por parte los mismos hacia los hijos (19).

Para algunos de los entrevistados, la obesidad se relaciona como un cambio en la imagen corporal o característica física visual en las personas, en relación con ello, Martínez et al. comentan que los participantes perciben la obesidad como una imagen corporal distorsionada y sentimientos de inferioridad. Con el mismo enfoque, Lerma et al. dicen: "El percibir al sobrepeso y a la obesidad como una cuestión de estética o una enfermedad depende del significado que se le da al exceso de peso mediante las pautas culturales e ideológicas que cada persona ha adquirido desde la infancia" $(20,21)$.

Los adolescentes percibieron en su mayoría a la obesidad como una enfermedad, un aumento de peso y aumento en los hábitos alimenticios, a lo cual Pérez et al., en algunas de las respuestas expresadas por los escolares entrevistados en su estudio cuando se les preguntó qué entendían y cómo percibían la obesidad, puede añadir: "enfermedad, relacionada con el exceso de comida y, por consiguiente, con el aumento de peso; se percibe como un padecimiento grave tal y como se aprecia en las siguientes expresiones: es mala para la salud, es la causa de la diabetes, es la causa de enfermedades cardiovasculares y puede provocar la muerte" (22). 
En cuanto a la prevención, tanto los padres y adolescentes señalaron como principal forma de prevenir la obesidad: una adecuada alimentación, excluyendo las comidas chatarras y los fritos. Lo cual coincide con Pérez et al., ya que explican que tanto hombres como mujeres mencionaron que consumir frutas y verduras es sinónimo: "de tener una alimentación nutritiva, tener una buena alimentación y consumirlas implica que no se van a caer los dientes". En los alimentos que se deben excluir dentro de la alimentación también se hallan similitudes al señalar papas fritas industrializadas, los refrescos y alimentos con altos contenidos de azúcares (22).

\section{Emociones}

Para esta investigación, los padres coinciden con los adolescentes sobre los hábitos que llevan a las personas a la obesidad, dentro de estos incluyeron el uso de dispositivos electrónicos. De forma análoga, Quintero et al. encontraron preocupante que las costumbres de la población infantil y juvenil de los países desarrollados se están replicando en Colombia en cuanto a permanecer muchas horas continuas, diurnas y nocturnas, frente a las máquinas, dejando de practicar juegos al aire libre. Por consiguiente, las familias coinciden en la percepción de que la obesidad y el cansancio en los ojos se deben a la dependencia al computador. Lo expuesto anteriormente, es confirmado por un padre que opinó sobre el tema: "A mi hijo casi no le gusta hacer tareas en el cuaderno, No sale de la alcoba, no se para del computador, se la pasa el día y noche pegado a ese aparato y ni siquiera baja a comer" (15).

\section{Sentimientos:}

Los resultados ante la socialización y percepción que tienen los entrevistados frente a las personas obesas fueron discriminación y bullying. Lo anterior, sumado a que Arias demostró en su investigación una relación entre bullying y el índice de masa corporal, puesto que el $40.76 \%$ del total de los escolares estudiados presentaron esta enfermedad, en donde el sexo femenino manifestó mayor probabilidad de ser víctima de bullying en comparación al sexo masculino, y teniendo en cuenta que Martínez, Flores et al., encontraron que "los adolescentes obesos experimentan el rechazo por sus pares o iguales en el ambiente escolar, son objeto de burla, maltrato y desprecio por parte de los compañeros que los hacen sentirse inferiores", esto contrasta con el resultado parcial de esta investigación, en donde las personas obesas fueron percibidas principalmente como nobles y tiernas, aunque, también se asocian a comportamientos negativos como personas depresivas, rechazadas, tristes, discriminadas y desorganizadas. Por lo tanto, se coincide con otros autores, como Lobera, Polo et al., cuando indican que las personas obesas fueron percibidas, con elevada frecuencia, como personas ansiosas, solitarias, vagas, apagadas, dependientes, miedosas, solas, infantiles, impulsivas y quisquillosas $(21,23,24)$.

\section{Conclusiones}

El estudio cualitativo reconoció los hábitos y creencias de padres y adolescentes respecto a la obesidad.

Intervenir a los padres y adolescentes permitió identificar las diferentes percepciones que tienen los grupos etarios frente al conocimiento, emociones y sentimientos que la obesidad genera en cada uno.

Las costumbres arraigadas en torno a la alimentación, sumado a las diferentes fuentes de información no confiables, pueden representar un factor de riesgo importante en la adopción de estilos de vida saludable. Así, la educación integral de los niños, jóvenes y adultos requiere estar rodeada de información confiable que logre garantizar un aprendizaje claro y pertinente.

En la actualidad, los padres reconocen que los adolescentes se encuentran con libertad para acceder a tecnologías virtuales, tanto en sus colegios como en sus hogares; según la literatura, el juego físico posee un papel fundamental en el desarrollo socioemocional, cognitivo y físico del niño, así como en la regulación de las emociones, el fortalecimiento de las relaciones sociales con los miembros de su familia y amigos, en la atención, en la resolución de problemas, en la creatividad y en el desarrollo de destrezas motoras gruesas y finas. Es decir, el juego físico ayuda en la salud en general; sin embargo, uno de los puntos que más se deben destacar, es su importancia para concientizar a la población en el adecuado uso de las tecnologías de diversión y ocio, además de apoyar el desarrollo del niño, especialmente en su aprendizaje y plasticidad cerebral, así como en su rendimiento académico.

En cuanto a la percepción que tienen los padres frente a las personas obesas, son evidentes los sentimientos de desconfianza, tristeza y rechazo generado por los mismos. De la misma manera, los padres las consideran personas con falta de conciencia y perezosas, puesto que, para la mayoría de ellos, la obesidad es ocasionada por el sedentarismo, y es una de las razones de burla y bullying. 
Teniendo en cuenta lo mencionado, el papel de los padres dentro del desarrollo nutricional del menor es determinante, por esta razón, es fundamental generar estrategias que permitan abordar tanto a los adolescentes como a los padres, con el fin de proporcionar un bienestar en la calidad de vida durante el desarrollo de sus hijos

\section{Declaración de conflictos de interés}

Los autores declaran no tener ningún conflicto de interés, derechos patrimoniales de la Corporación Universitaria Empresarial Alexander Von Humboldt y morales de los autores.

\section{Referencias}

1. Organización Mundial de la Salud (OMS). Obesidad y sobrepeso. [Internet]. [consultado 05 de marzo de 2018]. Disponible en: https://bit.ly/1hBxIq3

2. Encuesta nacional de la situación nutricional en Colombia 2010. [Internet]. [consultado 05 de marzo de 2018]. Disponible en: https://www.google.com/ url? sa $=$ t\&source $=$ web\&rct=j\&url=https://www. javeriana.edu.co/documents/245769/3025871/ Resumen Ejecutivo ENSIN 2010.pdf/160e9856006d-4a60-9da3d71606703609\&ved=2ahUKEwiL2 amvm5PnAhXQqFkKHReXDo8QFjAAegQIAhAB \&usg=AOvVaw3dF141nO7UfZ4QwaqmeL22\&csh $\mathrm{id}=1579558950419$

3. Escudero GV, Morales LV, Valverde C, Velasco JF. Riesgo cardiovascular en población infantil de 6 a 15 años con obesidad exógena. [Internet]. Rev Med Inst Mex Seguro Soc. 2014;52: 58-63 [citado 10 de abril de 2018]. Recuperado a partir de: https://www. medigraphic.com/pdfs/imss/im-2014/ims141j.pdf

4. Hurtado E, Macías R. Enfoque de la obesidad infantil desde la pediatría. [Internet]. Rev Med Inst Mex Seguro Soc. 2014; 52: 116-119. [citado 10 de abril de 2018]. recuperado a partir de: http:/www. medigraphic.com/pdfs/imss/im-2014/ims141s.pdf

5. Vera AM, Hernández BC. Documento guía alimentación saludable. [Internet]. [citado 18 de enero de 2018]. Recuperado a partir de: https://www. minsalud.gov.co/sites/rid/Lists/BibliotecaDigital/ RIDE/VS/PP/SNA/Guia-Alimentacion-saludable.pdf

6. Organización Mundial de la Salud (OMS). Enfermedades no trasmisibles. [Internet]. [citado 20 de agosto de 2018]. Recuperado a partir de: https:// www.who.int/es/news-room/fact-sheets/detail/ noncommunicable-diseases

7. Bastos AA, González R, Molinero O, Salguero del Valle A. Obesidad, nutrición y actividad física. [Internet]. Rev Int med cienc act fis deporte. 2005; 5:140-153 [citado 05 de enero de 2018]. Recuperado a partir de: http://cdeporte.rediris.es/revista/revista18/ artobesidad11.htm

8. Braguinsky J. Prevalencia de obesidad en América Latina. [Internet]. ANALES Sis San Navarra. 2002; 25 [citado 24 de marzo de 2018]. Recuperado a partir de: https://recyt.fecyt.es/index.php/ASSN/article/ view/5493/4534

9. Quintana EM, Salas MP, Cartín M. Índice de masa corporal y composición corporal con deuterio en niños costarricenses. [Internet]. Acta Pediátrica Mex. 2014;35: 179-189. [citado 24 de junio de 2018]. Recuperado a partir de: http://www.scielo. org.mx/scielo.php? script $=$ sci arttext\&pid $=\mathrm{S} 0186$ 23912014000300003\&lng=es\&tlng=es

10. Kain J, Corvalán C, Lera L, Galván M, Weisstaub G, Uauy R. Asociación entre el índice de masa corporal y la talla desde el nacimiento hasta los 5 años en preescolares chilenos. [Internet]. Rev Med Chile. 2011; 139: 606-612 [citado 25 de agosto de 2018]. Recuperado a partir de: https://scielo. conicyt.cl/scielo.php?script $=$ sci arttext\&pid $=\mathrm{S} 0034-$ 98872011000500007\&lng=es.

11. Dávila J, Gonzáles JJ, Barrera A. Panorama de la obesidad en México. [Internet]. Rev Med Inst Mex Seguro Soc. 2015; 53: 240-249 [citado 06 de enero de 2018]. Recuperado a partir de: http://revistamedica. imss.gob.mx/editorial/index.php/revista_medica/ article/viewFile/21/54

12. López GE, Perea A, Loredo A. Obesidad y maltrato infantil. Un fenómeno bidireccional. [Internet]. Acta Pediátrica Mex. 2008; 29:342-346 [citado 10 de julio de 2018]. Recuperado a partir de: https:// www.medigraphic.com/cgi-bin/new/resumen. cgi?IDARTICULO $=26124$

13. Núñez HP. Las creencias sobre obesidad de estudiantes de la educación general básica. [Internet]. Rev Educación. 2007; 41: 145-164 [citado 15 de febrero de 2018]. Recuperado a partir de: http://www.redalyc. org/pdf/440/44031110.pdf

14. Núñez HP, Campos N,Alfaro F, Holst L. Las Creencias sobre obesidad de niños y niñas en edad escolar y las de sus progenitores. [Internet]. Rev electrónica actualidades en Educación. 2013; 13:1-30 [citado 02 de marzo de 2018]. Recuperado a partir de: https:// www.redalyc.org/articulo.oa?id=44727049001.pdf

15. Organización Mundial de la Salud. Plan de acción para la prevención y el control de las enfermedades no trasmisibles en las Américas 2013-2019. [Internet]. [citado 10 de febrero de 2018]. Recuperado a partir de: https://www.paho.org/hq/dmdocuments/2014/ NCD-SP-low.pdf 
16. Quintero J. Munévar RA, Munévar FI. Ambientes escolares saludables. [Internet]. Rev. Salud pública. 2015; 17:229-241 [citado 20 de septiembre de 2018]. Recuperado a partir de: http://www.scielo.org.co/pdf/ rsap/v17n2/v17n2a07.pdf

17. Cabello ML, De Jesús D, Percepción de las madres de niños con obesidad sobre los hábitos alimenticios y sus responsabilidades en la alimentación de los hijos. [Internet]. Rev salud pública y nutrición. 2011; 12 [citado 08 de mayo de 2018]. Recuperado a partir de: https://www.medigraphic.com/pdfs/revsalpubnut/ spn-2011/spn111c.pdf

18. Olivares S, Bustos N, Moreno X, Lera L. Actitudes $\mathrm{y}$ prácticas sobre alimentación $\mathrm{y}$ actividad física en niños obesos y sus madres en Santiago, Chile. [Internet]. Rev chilena de nutrición. 2006; 3: 169-179 [citado 20 de enero de 2018]. Recuperado a partir de: $\quad$ https://scielo.conicyt.cl/scielo.php?script=sci arttext\&pid $=$ S0717-75182006000200006

19. Rodríguez A, Ruíz JP, Villagrán S, Martínez JM, Lechuga JL. La percepción del sobrepeso y la obesidad infantil por parte de los progenitores. [Internet]. Rev Esp Salud Pública 2012; 86: 483-494 [citado 07 de febrero de 2018]. Recuperado a partir de: http://scielo. isciii.es/pdf/resp/v86n5/03 originall.pdf

20. Martínez ML, Flores y, Rizo MM, Vázquez L. Percepciones de la obesidad de adolescentes obesos estudiantes del $7 \mathrm{o}$ al $9 \mathrm{o}$ grado residentes en Tamaulipas, México. [Internet]. Rev. Latino-Am, Emfermagem. 2010; 18: 1-7. [citado 25 de enero de 2018]. Recuperado a partir de: http://www.scielo.br/ pdf/rlae/v18n1/es_08.pdf

21. Lerma PS, Álvarez GC, Nazar DA, Mondragón R. Percepciones de adultos con sobrepeso y obesidad y su influencia en el control de peso en San Cristóbal de Las Casas, Chiapas. [Internet]. Estud. Soc. 2016; 47: 380-409. [citado 15 de enero de 2018]. Recuperado a partir de: http://www.scielo.org.mx/pdf/estsoc/v2425n47/0188-4557-estsoc-24-47-00380.pdf

22. Pérez SE, Mosqueda JI, Gabriela A, Juárez AG. Obesidad y alimentación: percepción de un grupo de niños y niñas de la costa de Oaxaca. [Internet]. Revista Infancias Imágenes. 2012; 11: 16-26. [citado 10 de mayo de 2018]. Recuperado a partir de: https://dialnet. unirioja.es/servlet/articulo?codigo $=4817206$

23. Arias GA. Bullying y su relación con la obesidad en escolares de séptimo año de educación básica de escuelas de la parroquia el sagrario de la ciudad de Loja en el periodo abril-noviembre. [Tesis]. [Loja, Ecuador]: Universidad Nacional de Loja; 2016.

24. Lobera I, Polo IM, González MT, Morales MT. Percepción de la obesidad en jóvenes universitarios y pacientes con trastornos de la conducta alimentaria. [Internet]. Nutr Hosp. 2008; 23: 226-233. [citado 13 de julio de 2018]. Recuperado a partir de: http://scielo. isciii.es/pdf/nh/v23n3/original5.pdf 